\title{
HE I LINES IN THE BE + K BINARY KX AND *
}

\author{
5. Stefl \\ Astronomical Institute, 25165 0ndřejov, Czechoslovakia
}

KX And (HD 218393) is a peculiar interacting binary with the period of about 38.9 days. This study is a first attempt to model the system as a double-line binary. The main results of the He I - line analysis are summarized.

The intensity tracings of sixty blue-violet spectra were used. All the spectra were obtained in the coudé focus of the Ondřejov $2 \mathrm{~m}$ telescope in the period 1972 - 1981. The original dispersion was 8 or $16 \mathrm{~A} \mathrm{~mm}^{-1}$. The phases of the observations were computed according to the ephemeris derived from the radial velocities of optical shell lines (Stefl et al. in preparation). Phase zero corresponds to the velocity maximum.

The centres of He I 4471 A and He I 4026 A were determined by means of the tracing paper using the mean of the direct and reverse image of the profile, and giving higher weight to the line wings to eliminate the strong profile deformation by the shell lines, particularly by the Ti II lines. Because of a low intensity and heavy line blending, the He I 4026 A - line measurements suffer from large errors. However, the results based on them qualitatively agree with all the following conclusions derived from the He I 4471 A measurements. The He I radial velocity varies with the same period as that of the shell lines but the radial-velocity curve is simple with the maximum in 0.0 and minimum in 0.5 . For comparison, the radial-velocity curve of the shell lines has two maxima in phases 0.0 and 0.35 and two minima in phases 0.2 and 0.65 (Fig. 1). The semiamplitude of the $\mathrm{He}$ I radial velocities is about $30 \mathrm{~km} \mathrm{~s}^{-1}$. The high value of the $\gamma$ - velocity $\left(-98 \mathrm{~km} \mathrm{~s}^{-1}\right)$ is worth noting. To check this result, the radial velocities published by Halliday (1950) were analyzed by the same way. In spite of the large scatter seen in the Halliday data, the curve agrees well with that obtained from the Ondrejor spectrograms.

The high $\gamma$-velocity of the He I lines can hardly be explained by the space motion of the star. In that case, the velocity of the absorption components of UV resonance lines relative to the star would be positive or zero (Stefl 1986). This contradicts their observed P-Cyg profiles. The systematically more negative measured radial velocities of the triplet He I lines could be caused by their asymmetry due to the forbidden components. However, test measurements of the $\mathrm{He} I$ 4471 A line in the theoretical spectra show that this effect can introduce a systematic error of about $-20 \mathrm{~km} \mathrm{~s}^{-1}$, but not more. It thus cannot explain the high negative $\gamma$-velocity of $K X$ And.

* Read by P. Koubsky. 
Fig. 1 Radial-velocity curve of a) He I 4471 A lines and b) the optical metallic shell lines.

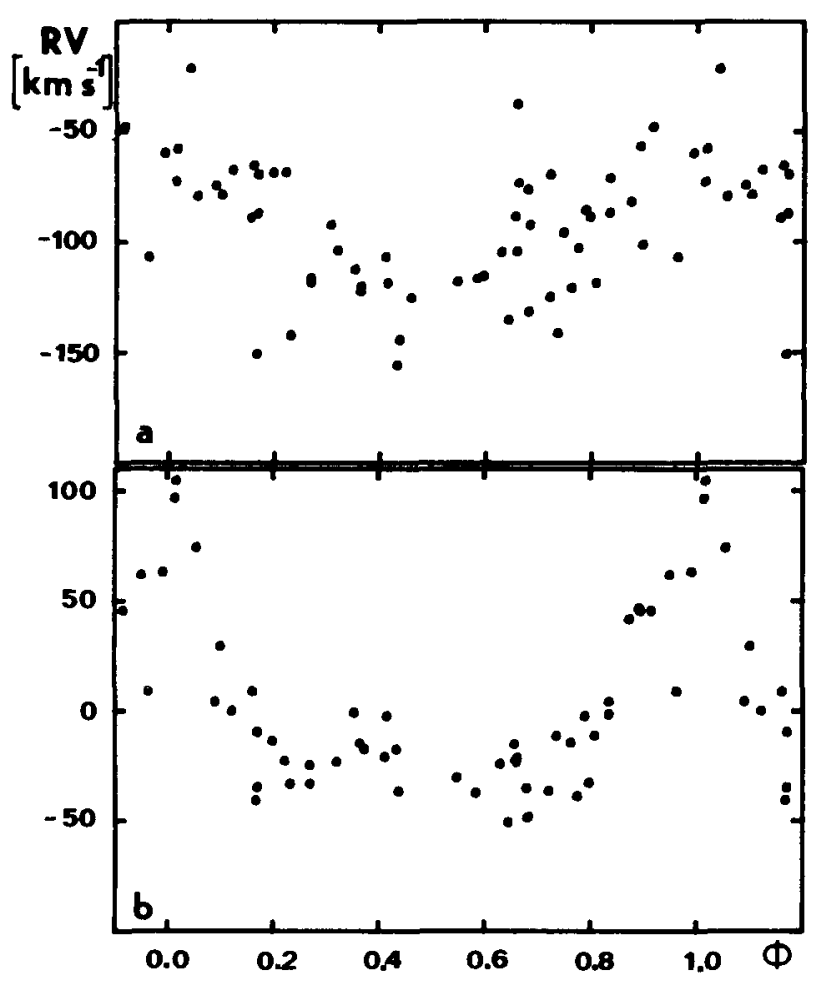

Using the semiamplitude of the radial velocity of the secondary component estimated from the infrared spectra, $K_{2}=100 \mathrm{~km} \mathrm{~s} \mathrm{~s}^{-1}$ (PoIidan 1976) and assuming that $\mathrm{He} I$ lines reflect the orbital motion of the primary, we can derive the values $M_{I} \sin ^{3} i$ and $M_{2} \sin ^{3} i$. From the absence of the photometric eclipses of the primary by the secondary presumably filling its Roche lobe and from modelling the accretion stream (using the computer code developed by Hadrava 1984) one gets $55^{\circ}<\mathrm{i}<70^{\circ}$

$8.2 M_{0}<M_{1}<11.6 M_{0}$, $2.4 M_{\odot}<M_{2}<3.5 M_{\odot}$ and assuming that the primary is a main-sequence star

$17300 \mathrm{~K}<\mathrm{T}_{\text {fff }}<27800 \mathrm{~K}$. However, the fitting of the He I 4471 A profiles by the synthetic ones in the phases when the shell spectrum is of the minimum intensity yield $\mathrm{T}_{\mathrm{eff}}$ about of $16000 \mathrm{~K}$. This difference and the above-mentioned $\gamma$ - velocity can be explained by the hypothesis that the $\mathrm{He} I$ lines originate in an expanding optically thick layer above the photosphere of the primary. Nevetheless, it is necessary to confirm the results presented here by a detailed study of the He I lines in the red region of the spectrum.

The author thanks Drs. P. Harmanec, P. Koubský, J. Horn, P. Hadrava and J. Krpata for obtaining the spectrograms used and for giving them kindly at his disposal, and Drs. P. Harmanec and I. Hubený for the valuable comments on the manuscript and data reduction.

\section{REFERENCES}

Hadrava, P. (1984). Bull. Astron. Inst. Czechosl. 35, 335. Halliday, I. (1950). Commun. David Dunlap Obs., no. 25, 149. Polidan, R. S. (1976). IAU Symp. 70, 401 and priv. com. to P. Harmanec. Stefl, S. (1986). IAU Symp. 122, in press. 


\section{DISCUSSION FOLLOWING STEFL}

\section{Doazan:}

On what lines is your $\mathrm{R} / \mathrm{V}$ curve based?

Harmanec:

The $\mathrm{R} / \mathrm{V}$ curve of shell lines mentioned here is based on the metallic shell lines. The $R / V$ curve of the shell lines varies indeed from line to line. The important point is that the $R / V$ range of the shell lines is over $100 \mathrm{~km} / \mathrm{s}$. Stefl measured $R / V$ on the wings of the HeI lines and he obtained a sinusoidal RV curve with $\mathrm{K}=30 \mathrm{kms}^{-1}$ only, which may better describe the orbital motion of the Be primary. 